\title{
A Hand-Centric Classification of Human and Robot Dexterous Manipulation
}

\author{
Ian M. Bullock, Student Member, IEEE, Raymond R. Ma, Student Member, IEEE, and \\ Aaron M. Dollar, Member, IEEE
}

\begin{abstract}
This work contributes to the development of a common framework for the discussion and analysis of dexterous manipulation across the human and robotic domains. An overview of previous work is first provided along with an analysis of the tradeoffs between arm and hand dexterity. A hand-centric and motion-centric manipulation classification is then presented and applied in four different ways. It is first discussed how the taxonomy can be used to identify a manipulation strategy. Then, applications for robot hand analysis and engineering design are explained. Finally, the classification is applied to three activities of daily living (ADLs) to distinguish the patterns of dexterous manipulation involved in each task. The same analysis method could be used to predict problem ADLs for various impairments or to produce a representative benchmark set of ADL tasks. Overall, the classification scheme proposed creates a descriptive framework that can be used to effectively describe hand movements during manipulation in a variety of contexts and might be combined with existing object centric or other taxonomies to provide a complete description of a specific manipulation task.
\end{abstract}

Index Terms-Dexterous, manipulation, robotic, human, hands

\section{INTRODUCTION}

$\mathrm{T}$ HE concept of dexterous manipulation has been discussed frequently in the research literature, particularly with respect to developing mechanical hands (robotic and prosthetic) that possess the immense functionality of the human hand. Indeed, the sophistication of the human hand and its coevolution with cognition is one of the most significant reasons for the amazing success of Homo sapiens in comparison to our most closely related primate relatives [1]. Each of our hands has as many controllable degrees of freedom (21) as both arms, wrists, and one leg combined. Furthermore, the majority of dexterous, in-hand manipulation tasks involve effort from multiple fingers, resulting in a large set of possible manipulation motions.

Partly due to the complexity associated with humanlevel dexterous manipulation, there have been relatively few research efforts devoted to understanding the full range of in-hand manipulation behaviors, evaluating an individual's ability to perform in-hand manipulation tasks as a means to benchmark against a physical impairment standard, or toward replicating the ability to perform a wide range of dexterous, in-hand manipulation tasks in engineered systems. This paper attempts to illuminate at least part of this space and lay the groundwork for future work in the area. To do so, we overview previous in-depth discussions of dexterous manipulation, detail scenarios in which dexterous, in-hand manipulation is desirable,

- The authors are with the Department of Mechanical Engineering and Materials Science, Yale University, New Haven, CT 06511.

E-mail: \{ian.bullock, raymond.ma, aaron.dollar\}@yale.edu.

Manuscript received 23 Nov. 2011; revised 23 Aug. 2012; accepted 27 Aug. 2012; published online 10 Sept. 2012.

Recommended for acceptance by A. Okamura.

For information on obtaining reprints of this article, please send e-mail to: toh@computer.org, and reference IEEECS Log Number TH-2011-11-0092.

Digital Object Identifier no. 10.1109/ToH.2012.53. present a thorough taxonomy for classifying in-hand manipulation, and use a number of example manipulation tasks to show how the taxonomy can be applied to common, real-world scenarios.

As we will discuss in the next section, there are a number of different ways to consider the decomposition of a manipulation task. These primarily consist of object- or environment-centric and hand-centric views. Consider an example task of unscrewing a cap on a bottle of water: An object-centric view of the task would prescribe a rotational motion of the cap along the major axis of the bottle combined with a force along that axis to lift the cap upwards. From a hand-centric perspective, this can be achieved in a number of different ways, such as through dexterous use of the thumb and forefinger with primarily within-hand motions, through a power grasp on the cap, and motion of the arm and wrist to unscrew, or through any number of additional possible methods that all enable the cap to be unscrewed. Due to the multitude of possible ways to execute the task with a given hand, as well as multitude of possible types of hands that can be used (such as different robotic or prosthetic hand designs), object-centric classification of manipulation tasks do not directly correlate with hand behavior or ability. Through the application of a hand-centric manipulation taxonomy, the steps taken by a given hand to execute a given task can be formally described, for instance, or the capabilities of different hands can be more directly compared.

We begin this paper with a discussion of the literature related to defining and describing dexterity and dexterous manipulation, both in the robotic and human domains (Section 2). We then discuss the general need for dexterity and contrast in-hand manipulation versus manipulation with a highly dexterous, redundant manipulator arm, and simple gripper (Section 3) as well as the semantics 
surrounding the definition of the "hand" or end-effector, particularly in tricky cases such as highly redundant continuum manipulators. Next, we present our handcentric manipulation taxonomy that subclassifies manipulation behavior according to the nature of the contact with external objects and the object motion imparted by the hand (Section 4)-The same taxonomy can be applied to all hands, end-effectors, manipulators, and terminal devices.

We then demonstrate examples of how the taxonomy might be utilized, including identifying and classifying a manipulation strategy (Section 5.1), evaluating capabilities of a robot or prosthetic hand (Section 5.2), informing design of everyday objects (Section 5.3), and analyzing activities of daily living (ADLs) (Section 5.4). We end with a discussion of the relationship to common robotic manipulation terminology and other classification schemes, including limitations of the taxonomy.

\section{BACKGROUND}

In this section, we begin with a discussion of how dexterity and dexterous manipulation have been previously described in the robotics literature (Section 2.1.1) and the human biomechanics, haptics, and rehabilitation literature (Section 2.1.2). In general, dexterous manipulation is typically referred to as manipulation involving one or more hands, and typically with motion occurring within the hand, sometimes referred to as "in-hand manipulation." Dexterity, however, is also used more broadly to describe other types of skillful physical motion, such as arm or leg movements. Interestingly, both the words "dexterous" and "manipulation" have roots that refer to hands ("manus"-latin for hand, and "dexter"-latin for right hand). In this paper, we use the term "dexterous manipulation" to refer to in-hand manipulation (sometimes using "dexterous, in-hand manipulation" to be redundantly clear).

The second section presents an overview of work related to previous efforts to create taxonomies for grasping and manipulation (Section 2.2), none of which, however, addresses a hand- and motion-based classification, instead focusing primarily on object-centric motion primitives, static grasp poses, and human-centric manipulation primitives, which could not be readily applied to other types of hands.

\subsection{Dexterity and Dexterous Manipulation}

\subsubsection{Related Work from the Robotics Literature}

In discussing robotic manufacturing, Hollerbach [2] and Wright et al. [3] discussed dexterity as a feature of assembly lines that would reduce the need for custom fixtures in different assembly tasks. The most dexterous hand, therefore, would be one that could serve as a general-purpose manipulator, capable of performing the most diverse set of operations in a manufacturing environment. In [3], a general, subjective dexterity spectrum is presented that attempts to compare various manipulators' dexterous capabilities as part of an attempt at a classification system that could optimally match manipulators with dexterous tasks. Okamura et al. [4] lays out the planning strategies, hardware design, and physical system parameters necessary to implement dexterous manipulation, noting that dexterous tasks require transitions between multiple grasp configurations to extend the kinematic limits of the system. Bicchi [5] focuses on the capabilities of multifingered robotic hands and suggests that the human hand's level of dexterity is currently still out of reach. His work on alternate hand designs argues that anthropomorphic solutions may not even be optimal for certain tasks [6].

These papers and others have put forth or utilized a definition of dexterity that varies considerably from one to the next. According to each, dexterity is:

- "(The) capability of changing the position and orientation of the manipulated object from a given reference configuration to a different one, arbitrarily chosen within the hand workspace," Bicchi [5].

- "(The) process of manipulating an object from one grasp configuration to another," Li et al. [7].

- "(When) multiple manipulators, or fingers, cooperate to grasp and manipulate objects," Okamura et al. [4].

- "(The) kinematic extent over which a manipulator can reach all orientations," Klein and Blaho [8].

- "Skill in use of hands" Sturges [9].

The primary difficulty in defining dexterity is differentiating it from general manipulation. Often, the utilized definition of dexterity is anthropocentric, denoting precision manipulation tasks primarily between fingertips and other small finger-like appendages. Indeed, the majority of "dexterous hands" that have been produced utilize anthropomorphic kinematics to attempt to emulate the dexterity of the human hand. The Utah/MIT Dexterous Hand [10], utilizing four tendon-based fingers with four degrees of freedom each, required 32 total antagonistic tendons to operate. Similar work has been done at the University of Bologna on the UB hand [11], and successive iterations, the most recent of which [12] uses compliant, elastic hinges to better emulate the underactuated, coupled behavior of human appendages. Other anthropomorphic hands of note include the Gifu hand [13], the DLR hand [14], the Robonaut hand [15], and the Karlsruhe humanoid hand [16]. One of the few nonfingered manipulators that provide "in-hand" prehensile manipulation is the turntable-based manipulator analyzed by both Bicchi and Sorrentino [17] and Nagata [18] that can manipulate objects through rolling.

\subsubsection{Related Work from the Human Hand Literature}

Researchers who study the human hand and hand impairment define and measure dexterity in various ways. Qualitative definitions often describe dexterity as involving fine, coordinated, efficient movements. An overview of various dimensions of dexterity and their perceived importance can be found in [19]. In his book on dexterity, Bernstein defines "the essence of dexterity" as "finding a motor solution for any situation and in any condition" [20], emphasizing the versatility of dexterous movements and the ability of the motor control system to adapt movements to challenging environmental conditions.

Quantitative clinical assessments of dexterity typically use completion time for a set of well defined, often 
repetitive, tasks [21]. A good score on these tests, however, does not necessarily mean all is well-subjects can use compensating movements to overcome problems from an injury or disability [22]. Despite their shortcomings, tests involving time to perform a small set of manual skills were found in one study to distinguish the level of independence of geriatric women better than any other single metric (with $\mathrm{p}<0.0001)$. Three skills alone were able to predict independence between three groups with 92 percent accuracy [23], better than other single factors such as mental status score, number of medical problems, or number of drugs taken.

Tests that give a more complete view of overall upper limb function are typically based on a therapist's qualitative description of how well the subject performs a set of tasks, but these assessment scores might vary from therapist to therapist [24]. There are very few existing tests, however, that involve substantial in-hand manipulation (or "fine manipulation skill"), with most simply involving pick-andplace operations under various conditions (e.g., object type, size, and mass, or location in the workspace).

Some more recently proposed assessment methods also use details of the subject's motion trajectory or force data. For example, Nowak [22] proposed that a measure of force coordination in precision grip could give a better measurement of stroke recovery. A study using a virtual pegboard insertion task suggests that grasping force profiles and collision forces with the virtual board could be used to assess sensory issues in stroke subjects [25].

\subsection{Related Classifications and Taxonomies}

\subsubsection{Human and Anthropomorphic Work}

While the authors were unable to find any extensive classifications that differentiate the full range of human manipulation behaviors from one another, a number of related works should be mentioned.

Substantial earlier effort has classified and categorized human grasp types, without thorough treatment of how those relate to manipulation of objects being grasped. A brief summary follows, but please see [26] or [27] for comprehensive review. Schlesinger [28] first categorized grasps into six types: Cylindrical, tip, hook, palmar, spherical, and lateral. These grasps are primarily defined by object geometry, but grasp choice is also determined by the task being performed. In 1956, Napier [29] suggested a scheme that would divide grasps into power and precision, but not all grasps fall cleanly into these two categories. Cutkosky then used observational surveys of professional machinists along with the previous work of Schlesinger and Napier to develop a more comprehensive grasp taxonomy [30]. This tree is first divided into power and precision grasps from left to right, and by shape and function down the tree.

A small number of successive taxonomies, built primarily with minor variations from the Cutkosky taxonomy, have been proposed. However, these have not yet been widely adopted. A preliminary investigation of grasp frequency in daily activities is presented in [31]. This study showed that a small subset of grasp types (six for one subject and nine for another) were used 80 percent of the time for the two subjects over the period examined.
Grasp synergies can offer an alternate, more continuous description of hand configuration, such as through an investigation of the principal components of hand poses used for grasping various objects (e.g., [32]). However, they are markedly different from the approach proposed in this work. This work, unlike the synergy approach, includes no description of hand configuration at all to allow application to and comparison of multiple hand types. For specific applications, it may be possible to use synergies or a grasp classification as a complement to the proposed taxonomy.

Iberall [26] provides a detailed discussion of oppositions possible with the human hand. These oppositions include three directions along which the human hand can apply forces, namely through pad, palm, and side opposition. Iberall then discusses various hand postures and grasp classification schemes within the framework of combinations of these fundamental human hand oppositions. There is also some discussion of dynamic grips such as the three-jaw chuck, which enable certain types of manipulation motion. Iberall's work is best suited to describing static poses or general opposition configurations for the human hand, but does not provide much description of hand motion, nor could it be easily applied to robotic manipulators. Some other taxonomies emphasize more dynamic behavior.

Although we were unable to find any manipulation taxonomies that can be applied to all manipulation motion and also apply to robotic hands, some related classifications should be discussed. Two closely related dynamic classifications are Elliott and Connolly's classification of intrinsic hand movements [33] and Exner's alternate classification [34].

Elliott and Connolly described three classes of withinhand (intrinsic) manipulation movements: Simple synergies, reciprocal synergies, and sequential patterns. In simple synergies, all digits involved move as one unit, such as while pinching or squeezing. In complex synergies, the fingers move together, but the thumb moves independently. In sequential patterns, the digits move independently in a repeated motion. This system defines a hand coordinate system using anatomical directions, which we also suggest for use when analyzing a human hand (see Section 4.3). One limitation of Elliott and Connolly's system is that it does not apply to all manipulation tasks-the authors note, for example, that flexor/extensor movements of individual digits are not included.

Exner's alternate classification has been used extensively in clinical settings, but simplifications made to ease clinical application may make it less suitable for research. Exner classified within-hand manipulation into five categories: Palm-to-finger translation, finger-to-palm translation, shift, simple rotation, and complex rotation. These five categories can be described in our taxonomy as translations or rotations about appropriate coordinate axes (shown later in Fig. 9).

Gentile's chapter in [35, Ch. 3] proposes a task classification scheme based on environmental context and function of the action. It differentiates tasks using criteria such as how the environment varies between trials. The taxonomy emphasizes the motor control complexity of a task and can be used to evaluate a patient's movement capabilities. However, the taxonomy does not provide any details about the manipulation itself. 


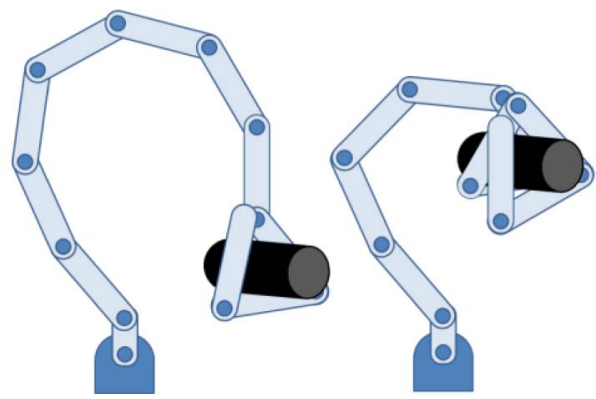

Fig. 1. A continuum-like manipulator example illustrates some of the challenges of differentiating the arm from the hand. Pettinato [41] suggest defining the hand by the linkages in contact with the object.

Since existing human classifications either only apply to certain subtypes of manipulation or do not provide detail about the manipulation movements, our proposed classification helps to overcome these limitations.

\subsubsection{Nonanthropomorphic and Robotic Work}

A number of works in the robotics literature have suggested that manipulation tasks can be broken down into a series of a small number of primitive motions, but they do not provide a hand-centric approach that can be applied to human and robot hands. Wright et al. [3] suggest that dexterous tasks can be decomposed into a set of primitive actions: Free motion of robotic fingers, acquiring an optimal grasp, turning a grasped object about an axis, and redistributing finger-tip forces. Other discussions of control systems for dexterous hands (e.g., [36]) also consider task decomposition into simpler, independent motions. Various previous work has used the term manipulation primitives to describe components of a manipulation motion. The term is usually applied to the steps used in a specific algorithm or by a specific robot, but work by Morris and Haynes [37] describes a more general definition based on motion in six possible degrees of freedom between two rigid bodies. Morrow and Khosla [38] later improved on the notation and described a general architecture for executing manipulation task primitives based on sensorimotor primitives defined for a specific robot. Michelman [39] describes a small set of primitive manipulation motions for the Utah/MIT hand, and then explains how complex tasks can be described as a series of these fundamental motions. Task steps are specified in terms of the force and position control required. While Michelman's task description shares some similarities with our final motion sub-classification (Section 4.3), the manipulator primitive approach differs from ours in that cannot easily be applied to compare multiple manipulators of different types. In general, the object motioncentric approach taken is different from our more handcentric taxonomy.

A much different but related classification is the taxonomy of haptic disassembly tasks [40]. The proposed taxonomy classifies tasks according to task type and type of force or torque required. The force classification differentiates between tasks where the force is aligned with the motion, such as pressing a button, and those where the force is not aligned, such as sanding a surface. Torque is differentiated by whether the torque axis passes through

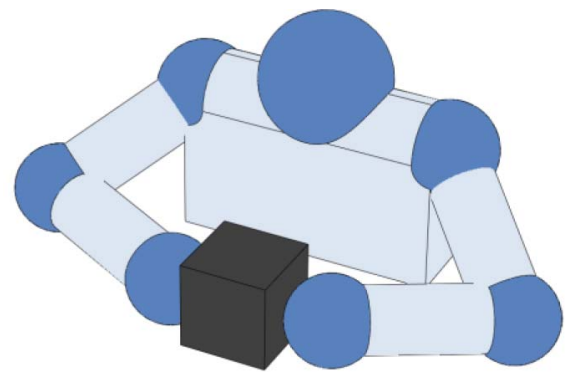

Fig. 2. Bimanual manipulation is nearly identical to precision in-hand manipulation on a larger size scale.

the grip space, expressing the difference between turning a screwdriver and a steering wheel. However, the task type classification focuses on haptic simulation constraints, making it less appropriate in other contexts. For example, a significant force category is used because of the limited force capabilities of haptic devices, and a tool assisted category is used because the shaft of a haptic device often allows easier simulation of tasks in this category.

The substantial previous work described above has focused primarily on static grasp posture classification, object-centric or environment-centric classification, or a human-centric treatment of only a subset of possible manipulation movements. No hand-centric taxonomy was found that can easily apply to both a robot and human hand. Our present hand and motion centric taxonomy addresses these limitations by being applicable for any type of manipulation performed by a hand-type manipulator.

\section{ARM VERSUS HAND DEXTERITY}

To understand and classify hand-based dexterous manipulation, it is important to define what is meant by a "hand" (Section 3.1), as well as discuss when in-hand manipulation is useful compared to arm-based manipulation (Section 3.2).

\subsection{Differentiating Arm and Hand}

The above descriptions and definitions of dexterity are sufficiently general such that there is not a clear differentiation between dexterity provided by the manipulator "arm" and the end-effector or "hand." In manufacturing and anthropomorphic systems, the arm is generally a multidegree of freedom linkage-based system (often including a wrist), to which the end-effector, or hand, is attached. While this type of system exhibits clear differentiation between the arm/wrist and hand, this framework does not necessarily apply to all systems. In his description of a continuum-like manipulator, Pettinato and Stephanou [41] suggest that the "hand" can be defined as the set of linkages in contact with the object, in which case the task and object would define the separation between the two (Fig. 1).

Consider another "handless" system where multiple manipulator arms without end-effectors work together to manipulate an object, such as in common "bimanual" tasks (Fig. 2) (e.g., [42]). If these multiple manipulators were situated together as the end-effector of another manipulator arm, they might be naturally viewed as the "hand." Accordingly, the scale of the object/task related to that of the overall system biases the interpretation of whether a 


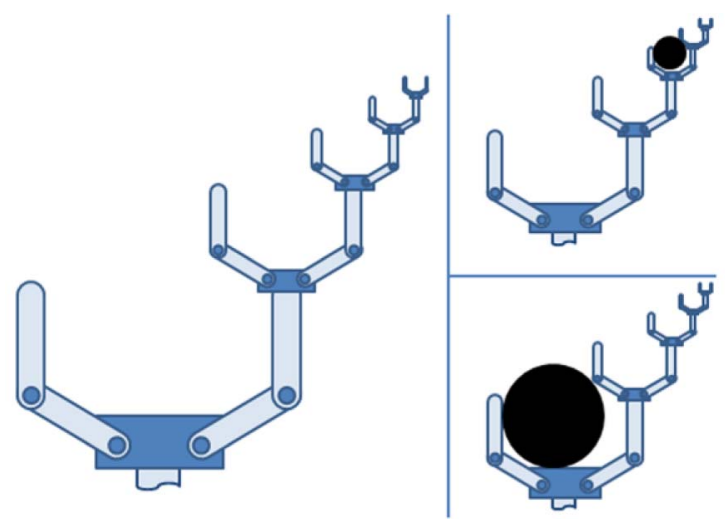

Fig. 3. Fractal manipulator showing different scales of manipulation. On one scale, the "fingers" grasping the object become part of the "arm."

mechanism is considered an "arm" or a "hand." Taking this illustration to an extreme, consider a "fractal" manipulator, consisting of a series of successive smaller graspers anchored to the tip of one of the previous stage's fingers (Fig. 3). For a given object or task, there is a particular grasper (i.e., matched finger pair) that is most appropriate based on the size of the object to be manipulated. This pair then determines the division between what is considered the "hand" (i.e., grasper) and the "arm" that imparts the manipulation capability.

Due to the blurry line between arm and hand, the taxonomy described in Section 4 does not rely on a strict definition. Rather, the user can define the "hand" themselves, or can simply focus on the location of the contact with the external object.

\subsection{Arguments for Arm- versus Hand-Based Manipulation}

Due to the extensive capabilities of the human hand, its level of functionality has proven to be extremely difficult to emulate. Mechanically, it is challenging to incorporate a large number of articulated degrees of freedom and the subsequently required number of actuators and transmission components. From a controls perspective, the lack of highquality, robust, and readily available sensing technologies to provide precise and high-bandwidth feedback about the nature of the contact conditions and the internal states of the mechanism result in imprecise position and force outputs.

Given these substantial challenges, why is in-hand manipulation truly needed? From an object-centric view of the problem, is not it sufficient to arbitrarily position and orient the object within some reasonable workspace volume, while being able to apply useful forces through the object? Since this capability can be accomplished with a redundant manipulator arm and a simple gripper, are the complications associated with implementing hand dexterity for in-hand manipulation worth addressing?

We lay out a few arguments related to this question below and briefly summarize them in Table 1 .

\subsubsection{A Dexterous Arm with a Simple Gripper Is Sufficient}

If dexterous manipulation can be thought of from an objectcentric perspective, it is desirable to have the ability to place the object in an arbitrary set of positions and orientations
TABLE 1

Considerations for/against In-Hand Dexterity

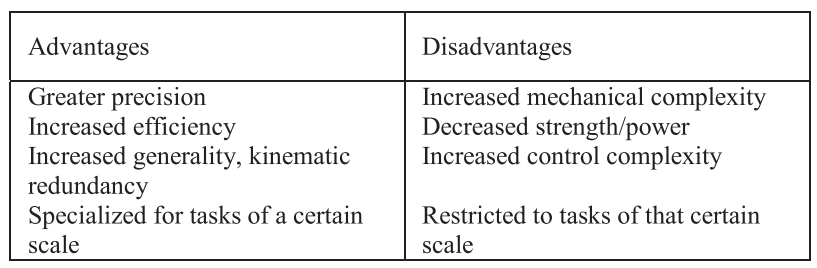

(six degrees of freedom) within some workspace while retaining the ability to do "something useful" with the object in that configuration-for instance, being able to write with a pen, apply force to insert and turn a key, and so on.

According to this (somewhat limited) description of task functionality, a simple gripper sufficient to stably grasp a wide range of objects combined with a highly dexterous arm should be able to accomplish most of the tasks needed. Even in cases where limitations in the starting grasp configuration of the object in the simple gripper conflicts with the desired task goal, the object might be regrasped at a different orientation [43] to compensate for the lack of inhand manipulation ability and accomplish the task. Given the greater simplicity in this approach, where the hand is for simple grasping and the arm is for manipulation and external force application, a dexterous arm and a simple gripper is sufficient and appropriate for many manipulation tasks. Simple grippers may be particularly well suited for tasks where high robustness and low cost are needed, or when less overall dexterity is required, such as when the environment and task are more structured.

\subsubsection{A Dexterous End-Effector Can Make Up for Limitations in Arm Functionality}

In practice, there are a number of situations in which the manipulator arm is not sufficiently functional to enable all desired manipulation tasks to be executed. For simple grippers, the configuration space of the object is limited by the configuration space of the arm, as the hand only serves to assemble the object to the arm. At joint limits and arm singularities, the possible motions of the object become very limited without a dexterous end-effector (Fig. 4). The presence of obstacles also places constraints on the set of possible arm configurations, effectively creating virtual

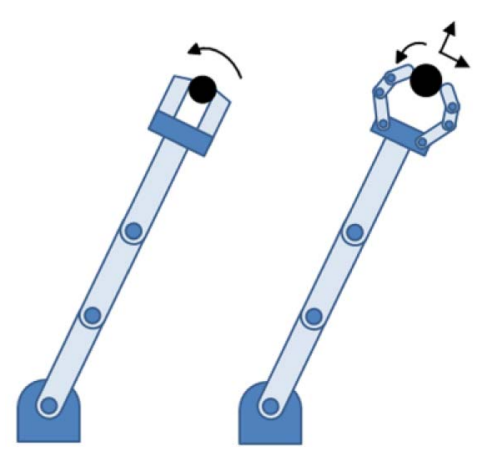

Fig. 4. Joint limits can restrict the capabilities of a dexterous arm. 


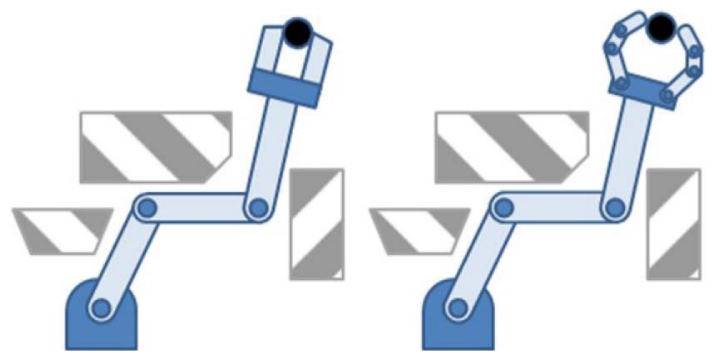

Fig. 5. Obstacles impose virtual joint limits on dexterous arms.

joint limits. In-hand manipulation can then replace some of the lost ability due to these constraints (Fig. 5). Hand dexterity can greatly increase the workspace of the system distal to the "arm," which is particularly useful at arm singularities or in the presence of obstacles.

At the end-effector level, a dexterous hand primarily adds kinematic redundancy that might otherwise be accomplished by adding active joints to the manipulator arm. In scenarios, where a power grasp is required to secure the manipulated object, the dexterity of the hand is greatly reduced, and even a dexterous hand's function becomes comparable to that of a parallel-jaw gripper. In these cases, additional arm dexterity is more beneficial than hand dexterity.

However, for objects and tasks at scales, where precision grasps are sufficient for force closure, additional hand dexterity can sometimes achieve the goal state entirely within the hand subsystem without any additional action from the arm. By actuating only the smaller finger mechanisms, a dexterous hand can enable increased precision and speed compared to movements from a larger arm. Indeed, in human manipulation, the arm (or wrist) is often braced on a surface to decouple the hand and the arm in precision tasks, such as writing with a pen.

\subsubsection{Manipulation with a Dexterous End-Effector Is Sometimes More Appropriate for a Given Task}

The concentration of kinematic redundancy and complexity in the end-effector as opposed to other portions of the arm has benefits for certain tasks, particularly related to the scale of motion and precision required. Most precision tasks require only small degrees of motion, making it inefficient or inappropriate to utilize whole-arm movements. Manipulations with a dexterous hand can reduce the energy required to accomplish the task, due to the lower inertial loads that must be moved. Related to this point, the use of a dexterous end-effector for fine manipulation reduces the magnitude of the feedback gains required in the control system for good performance as opposed to a full arm, which in turn increases the safety of the system, increases mechanical adaptability and compliance (useful for passively accommodating small positioning and alignment errors), and decreases electrical power usage.

When handling tools or objects that need to have certain features exposed (e.g., the "business end" of a tool or implement), a dexterous end-effector allows the object to be reoriented within the hand from the initial grasp, such as switching from a fingertip grasp to a power grasp. A simple gripper and manipulator arm would be forced to release the

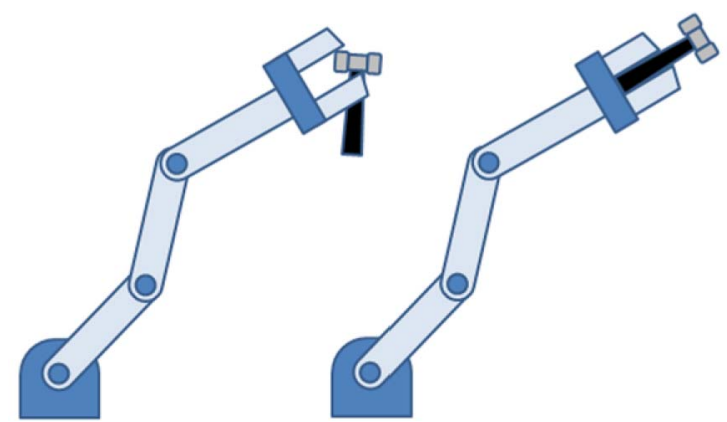

Fig. 6. Reorientation of tools within the hand is difficult, if at all possible, with simple grippers.

object and regrasp it in a more appropriate configuration (Fig. 6), which may not be desirable in certain scenarios. A dexterous manipulator could allow for reorientation to occur while the object remains in a stable grasp within the hand, which may be necessary or advantageous.

\section{Classifying Dexterous Manipulation}

In this section, we first define the terms relevant to our proposed taxonomy, then present the taxonomy and a brief description of how it can be utilized (Section 4.2), followed by a discussion of possible subclassifications (and the presentation of one based on the direction of motion with respect to the hand) (Section 4.3), and finish by discussing how complex tasks might be decomposed (Section 4.4).

\subsection{Definition of Terms}

The following text provides the definitions of the terms used in the taxonomy for each subclassification, with corresponding diagrams in Fig. 7. Since this taxonomy is "hand centric," the majority of the terms describe the nature of the hand's interaction with external objects:

- Contact. The hand is touching any external solid or liquid aside external to the hand itself.

- Prehensile. Hand contact with an object is prehensile if it cannot be reasonably represented by a single contact point ("virtual finger" [28]). Equivalently, contact is prehensile if the contact forces from the hand alone can stabilize the object without need for external forces such as gravity or from "ground."

- Motion. The hand is moving with respect to a body coordinate frame, such as the torso. A "world frame" is not used to distinguish from cases where the whole body is moving, such as on an escalator.

- Within Hand. Motion occurs within the hand, and therefore parts of the hand, such as the fingers, are moving with respect to a frame fixed to the base of the hand. As a counter example, motion does not occur within the hand in cases where the hand is purely securing the object to the arm, such as in power grasps. In these, the motion of the contacted object is primarily affected by the arm or other parts of the body, but not by the hand.

- Motion at Contact. The hand is significantly translating or rotating the object (or a combination of both) with respect to a frame affixed to the contact 


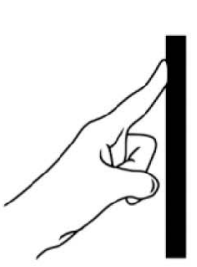

\section{Contact}

Hand is touching an external object or the environment.

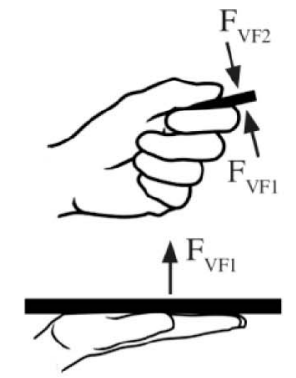

Prehensile

Action of hand on object must be described with more than one virtual finger.

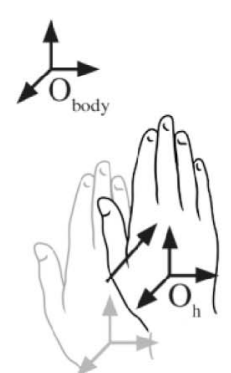

Motion

Any part of the hand moves relative to body fixed frame.

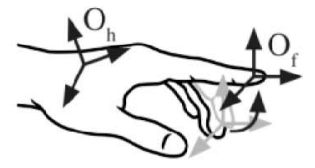

Within Hand

Points on the hand are moving relative to the hand base frame.

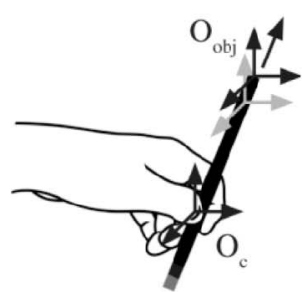

Motion at contact Object reference frame moves relative to contact point frame(s).

Fig. 7. Explanation of important terms in the manipulation taxonomy.

location(s) on the hand. Most manipulations involve some small contact changes due to skin deformation (such as writing with a pen), but these can be considered unintentional and not significant. Cases where the contact point stays the same, but the object is being pivoted, would be classified as "motion at contact," because points on the object move relative to the fixed contact frame(s).

- Dexterity. Loosely defined as the complexity of the manipulation task, or the skill involved in performing a manipulation task.

- Precision. Loosely defined as the accuracy able to be attained with the given configuration and category.

\subsection{Hand-Centric Manipulation Taxonomy}

Fig. 8 presents our manipulation taxonomy ${ }^{1}$ (with terms defined in Section 3.1). The presented subclasses are meant to apply to "instantaneous" or "discrete" hand movements, rather than longer time sequences (see Section 4.4 for further discussion). While we use human hands to illustrate each subclass, this taxonomy applies to any "hand." In creating this classification, we take a hand-centric view of the problem, as opposed to an object-centric view. The taxonomy, therefore, focuses on what the hand is doing during execution of the manipulation task. Example tasks for each subclass are given in Table 2 .

As described in Section 1 of this paper, there might be many ways for a hand to execute an object-centric task description (e.g., a low-friction knob could be turned with a single finger as a nonprehensile task, or with multiple fingers as a prehensile task). However, because the proposed classification consists of small set of yes/no criteria (e.g., contact or no contact), a given hand movement has only one possible classification on the taxonomy tree (except for complex tasks in which the hand is simultaneously doing multiple things, described in more detail in Section 4.4). An object-centric classification similar to [38] might be used in addition to the hand-centric taxonomy to give a more

1. A preliminary version of this taxonomy was presented in [52]. The version presented here has been modified to include an additional class of manipulative movements that we had not previously identified, these being ones where there is motion at contact without motion of the hand (subclasses 5, 7, 9, and 11). Numbering of the subclasses has also been added. thorough description of both what the hand is doing as well as what is being done to the object it is manipulating.

Note that in addition to being hand centric, this is also a motion-centric view of manipulation tasks, as opposed to a force-centric view (such as [40], as described above). However, the two are related by the Jacobian of the manipulator so that motions can occur in directions in which forces can be applied and vice versa.

\subsection{Further Subclassification}

Further subcategories could be added depending on the specific application of the taxonomy. For categories where there is contact but no motion is occurring (e.g., classes 4 and 6]) a grasp taxonomy ([27], [30]) might be directly applied, for instance, to further describe the nature of the hand posture.

For categories in which motion is occurring, perhaps the most natural subclassification would be based on the direction of motion with respect to the hand. Fig. 9 shows such a subclassification (which could be applied to any subcategory), applied to the prehensile, withinhand manipulation category (classes 14 and 15) This is the subcategory that most would consider the best example of "dexterous manipulation." Classification is done by the principal axis of motion with respect to a coordinate frame affixed to the back of the hand. Each movement subcategory ("no motion at contact" and "motion at contact") is, therefore, expanded to three rotational and three translational movements with respect to this coordinate frame (plus some positional offset). When classifying the human hand, radial-ulnar, distal-proximal, and dorsal-palmar axes can be substituted for the $x-, y-$, and $z$-axes, respectively. Movements significantly askew from the hand axes might be described by either the closest aligned axis or a combination of movements involving the principal axes. Note that dexterous motions in a few directions are difficult for the human hand to accomplish given its kinematic abilities (e.g., $x$-axis translation and rotation about the $y$-axis).

Other subcategorizations could also be made, such as by the degrees of freedom the object can actively be manipulated in (similar to [38]), or related to the forces used, similar to [40]. 


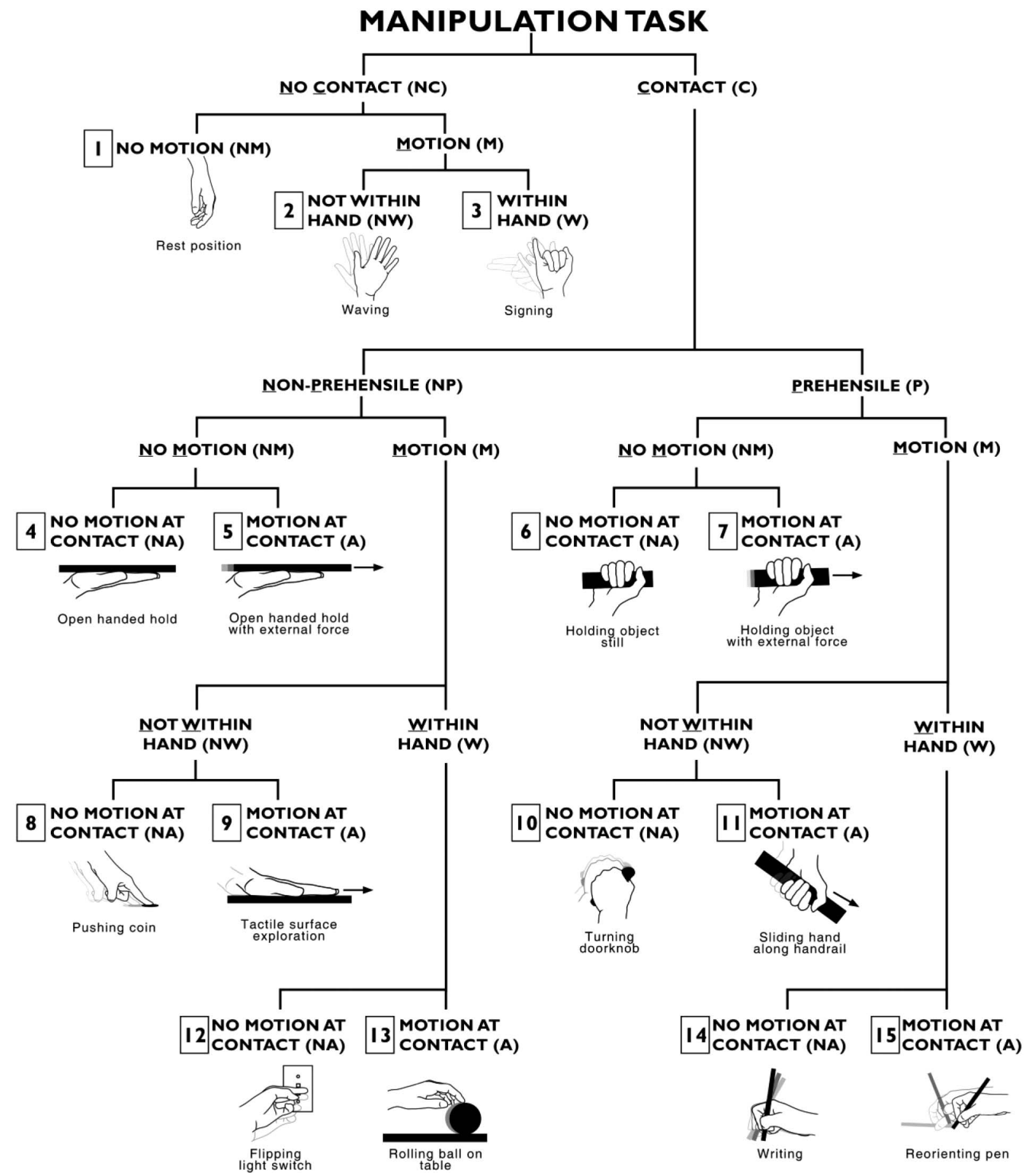

Fig. 8. Manipulation taxonomy. Any type of human or robotic manipulation task can be classified, according to the simple criteria defined in Fig. 7. Example tasks are given for each leaf of the tree.

\subsection{Composition of Complex Tasks}

The taxonomy presented above provides a structured way of classifying simple manipulative movements. We propose that complex tasks be described as a composition of smaller subclasses. Three major types of complex manipulation tasks that should generally be split into separate subtasks come to mind: time-separated sequences, simultaneous bimanual tasks, and simultaneous within-hand tasks.

Time-separated motions, such as a long sequence of movements to accomplish an overall goal, should be classified as the sum of the discrete subcomponents of the manipulation process. For instance, picking up a pen and writing with it might be decomposed into three subtasks: Lifting the pen from the table (C P M NW NA 10) rotating the pen into the writing position (C P M W A 15), and writing with it (C P M W NA 14). Detailed examples of additional time-separated motions are given in Section 5.1.

Bimanual tasks, where both hands are in use and required to perform a single task, might be classified by sum of the individual tasks being performed by each hand. 
TABLE 2

Common Manipulation Tasks

\begin{tabular}{|c|c|}
\hline Category & Example Tasks \\
\hline \multirow{3}{*}{$1 \mathrm{NC} \mathrm{NM}$} & Hand in rest position \\
\hline & Blocking out sun \\
\hline & Raising a hand \\
\hline \multirow{2}{*}{$2 \mathrm{NC} \mathrm{M} \mathrm{NW}$} & Waving \\
\hline & Hand swinging (e.g. while walking) \\
\hline \multirow{2}{*}{$3 \mathrm{NC} \mathrm{M} \mathrm{W}$} & Signing \\
\hline & Preshaping \\
\hline \multirow{2}{*}{4 C NP NM NA } & Open handed hold \\
\hline & Leaning on table \\
\hline 5 C NP NM A & Open handed hold with external force \\
\hline 6 C P NM NA & Holding object still \\
\hline 7 C P NM A & Holding object with external force \\
\hline \multirow{3}{*}{8 C NP M NW NA } & Sliding coin across table \\
\hline & Pushing large box \\
\hline & Pushing a push bar door open \\
\hline \multirow{2}{*}{9 C NP M NW A } & Tactile surface exploration \\
\hline & Sliding finger across large touch screen \\
\hline \multirow{5}{*}{10 C P M NW NA } & Turning doorknob \\
\hline & Wiping surface (e.g. with a sponge) \\
\hline & Pushing broom \\
\hline & Knife chopping \\
\hline & Power turn (e.g. loosening large lid) \\
\hline \multirow{2}{*}{$11 \mathrm{C} P \mathrm{M}$ NW A } & Sliding hand along handrail \\
\hline & Doing a pull-up (with hand rotation) \\
\hline \multirow{3}{*}{12 C NP M W NA } & Flipping light switch \\
\hline & Pushing button \\
\hline & Pushing piano key \\
\hline \multirow{2}{*}{13 C NP M W A } & Rolling ball between hand and table \\
\hline & Non-prehensile knob/lid turning \\
\hline \multirow{7}{*}{14 C P M W NA } & Writing \\
\hline & Precision lid turning \\
\hline & Inserting key \\
\hline & Precision lift \\
\hline & Cutting with $\mathrm{x}$-acto ${ }^{\circledast}$ knife \\
\hline & Using scissors \\
\hline & Squeezing sponge \\
\hline \multirow{4}{*}{15 C P M W A } & Reorienting pen \\
\hline & Precision to power grasp transition \\
\hline & Regrasping (within hand) \\
\hline & One handed touchscreen phone use \\
\hline
\end{tabular}

When both hands are used to perform independent tasks, each hand can be classified separately.

Tasks where a single hand performs two or more simultaneous functions (e.g., pulling a hand drill trigger, thumb typing on smartphone, using calipers, writing on a chalkboard, and so on) are some of the most dexterous tasks regularly performed. These typically involve part of the hand stably grasping the object while another part (often the thumb or index finger) moves independently. We propose that these types of tasks be treated as the sum of the distinct subtasks being performed. For

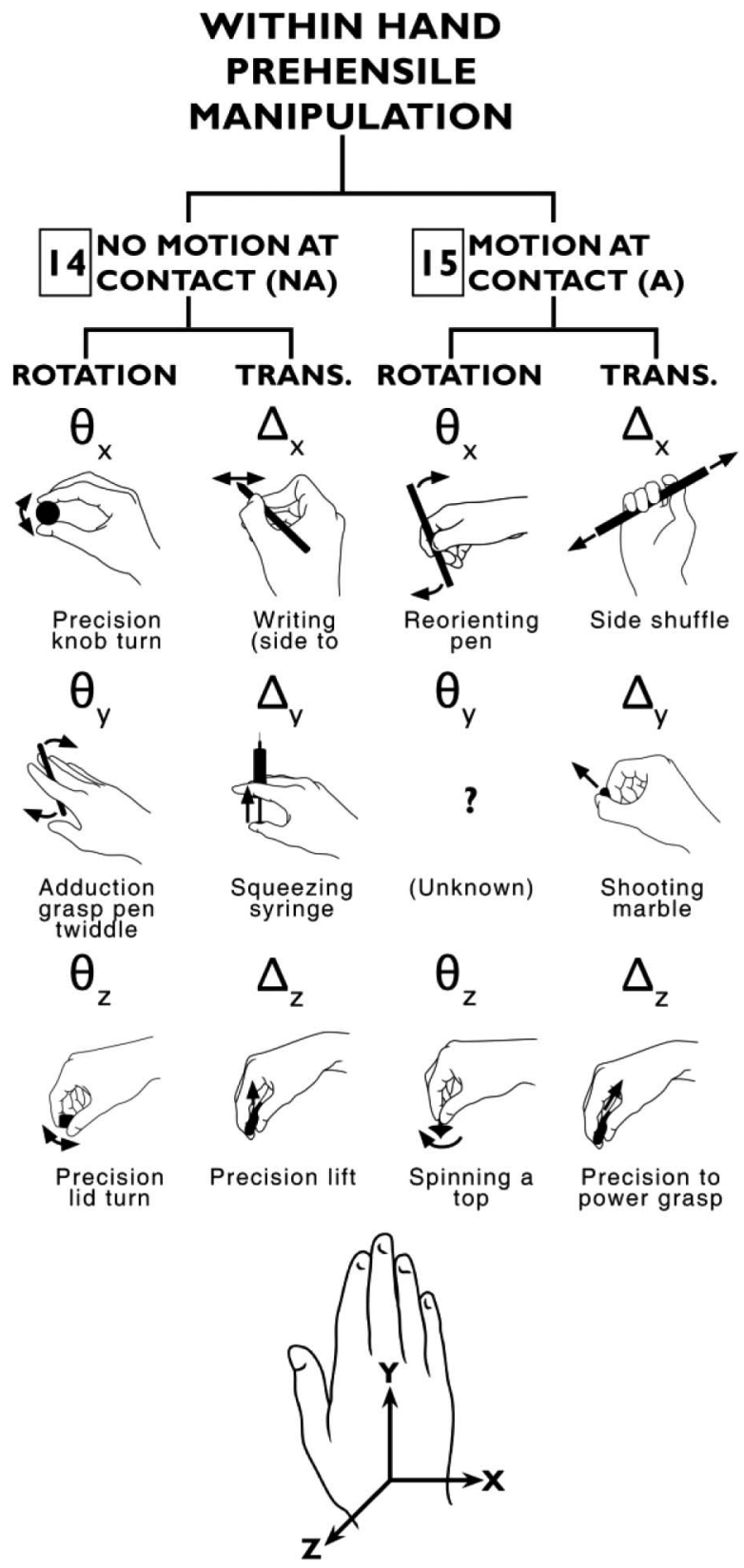

Fig. 9. Dexterous subclassification. Tasks are classified by rotations and translations along hand coordinate axes. Note that if the human hand is being analyzed, the standard anatomical hand axes can be substituted. Ulnar and radial can be used to specify $x$-axis directions, distal and proximal for the $y$-axis, and dorsal and palmar for the $z$-axis.

example, the task of pulling the trigger on a power drill could be categorized as a prehensile, no motion task 6 (grasping and holding the drill handle) combined with a nonprehensile/motion/within hand task 12 (index finger compressing the trigger). Thumb typing on a cell phone would be similarly considered as the sum of a prehensile, no motion task 6 (holding the phone with the palm and fingers) combined with a nonprehensile/ motion/within hand task 12 (thumb pressing the keys). Writing on a chalkboard, where a force is being applied to the board (to maintain contact) and the chalk is being moved along the surface of the board would be considered as the sum of two prehensile/motion/within 

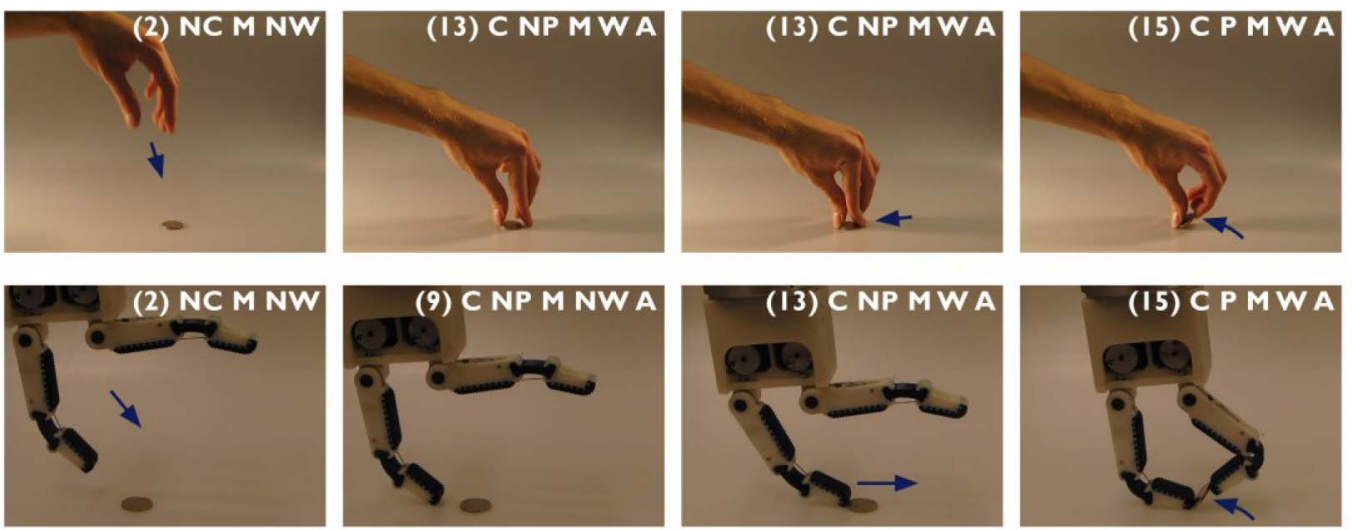

Approach

Table contact

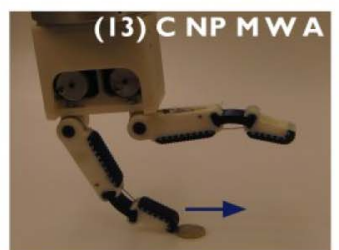

Coin sliding

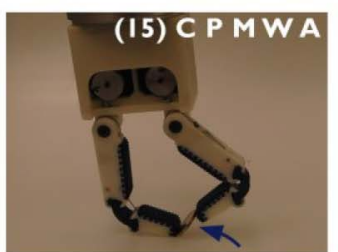

Coin rotation
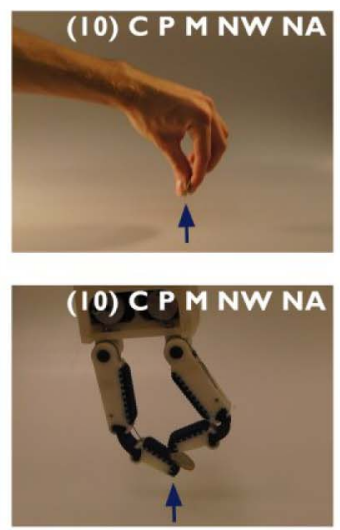

Pickup

Fig. 10. Illustration of how human manipulation can be analyzed as inspiration for robotic manipulation strategies. The task shown is a coin pickup using the human hand and a two-fingered robot hand [44].

hand tasks 14 as applying force to the board and moving the chalk both require actuation in each direction.

For certain applications of the taxonomy, it may be desirable to simply use a single classification for the overall hand rather than a composition, such as when looking at the time-varying changes of the taxonomy classification during a complex task (see, e.g., Section 5.4). This approach tends to simplify the classification process but may provide less detail about the manipulation. Thus, it can be left to the user to decide whether or not to break up the tasks when the hand is performing multiple functions.

\section{Taxonomy Applications}

In the following, we provide examples of how our taxonomy can be applied to analyze a manipulation task or series of tasks, evaluate a hand design, or be applied to product design or design for assembly for ergonomic purposes.

\subsection{Identifying a Manipulation Strategy}

One potential use of the taxonomy is to identify and help classify a manipulation strategy for executing a certain task Fig. 10(top) shows the analysis of a human picking up a coin from a table. In this figure, the subclassification of Grasping small objects placed on flat surfaces is a particularly difficult task because the surfaces of the object available for grasping are small and the table presents a contact barrier. An approach that mimics the human strategy for this task has been implemented with great success (as shown in the bottom of Fig. 10) [44].

Specifically, the robot hand approaches from one side and first slides the coin across the table, while the human hand approaches the center of the coin and brings both the thumb and the index finger in toward the coin after table contact is made. The final stages of coin rotation and pickup are very similarly executed by both systems. The main classification difference results from the human hand using preshaping ( $\mathrm{C} \mathrm{NP} \mathrm{M} \mathrm{W} 13$ ) leading up to the table contact, while the robot hand does not use any within-hand motion at this point (C NP M NW A 9)

\subsection{Robot or Prosthetic Hand Analysis}

Since the proposed taxonomy provides a descriptive vocabulary for discussing dexterous manipulation and different manipulation strategies, it can also be applied to a general discussion of the dexterous capabilities of a robot hand or arm-hand system and to compare different hands. Fig. 11 illustrates the type of analysis that can be performed, using a simple three-fingered hand as an example. This example hand has two revolute joints in each two-link finger but cannot rotate the fingers at the base. This allows only certain types of within-hand manipulation to occur.

The subclassification helps to discuss in detail which types of manipulation are easier or harder to accomplish with this hand. Most manipulations in the plane of the two aligned fingers are easier, such as $X$ and $Y$ translation and $Z$ rotation with no motion at contact 14 . Movements with motion at contact 15 require two fingers to stably grasp the object (the coplanar fingers) while the perpendicular finger rotates or slides the object. Some motions are simply not possible at all due to the kinematic structure of the hand. The same type of analysis can be used to discuss the within-hand manipulation capabilities of many other robot hands or even of the human hand.

This type of analysis is somewhat similar to a formulation of a basic set of manipulation primitives such as is done in [39]. However, applying the taxonomy does allow some additional analysis. In addition to this within-hand analysis, we can use the taxonomy to differentiate between different types of manipulation the overall robotic system can achieve. For example, one could analyze how well suited the system is to nonprehensile manipulations of certain types, or how well the arm-based dexterity complements the within-hand dexterity.

\subsection{Applications for Design}

The taxonomy can also be used to inform the design of parts and products that humans might manipulate. Applications include providing a helpful descriptive vocabulary during the design process, a means of analyzing the difficulty accomplishing a certain task or interacting with a certain interface, or to investigate the variety of movements used 


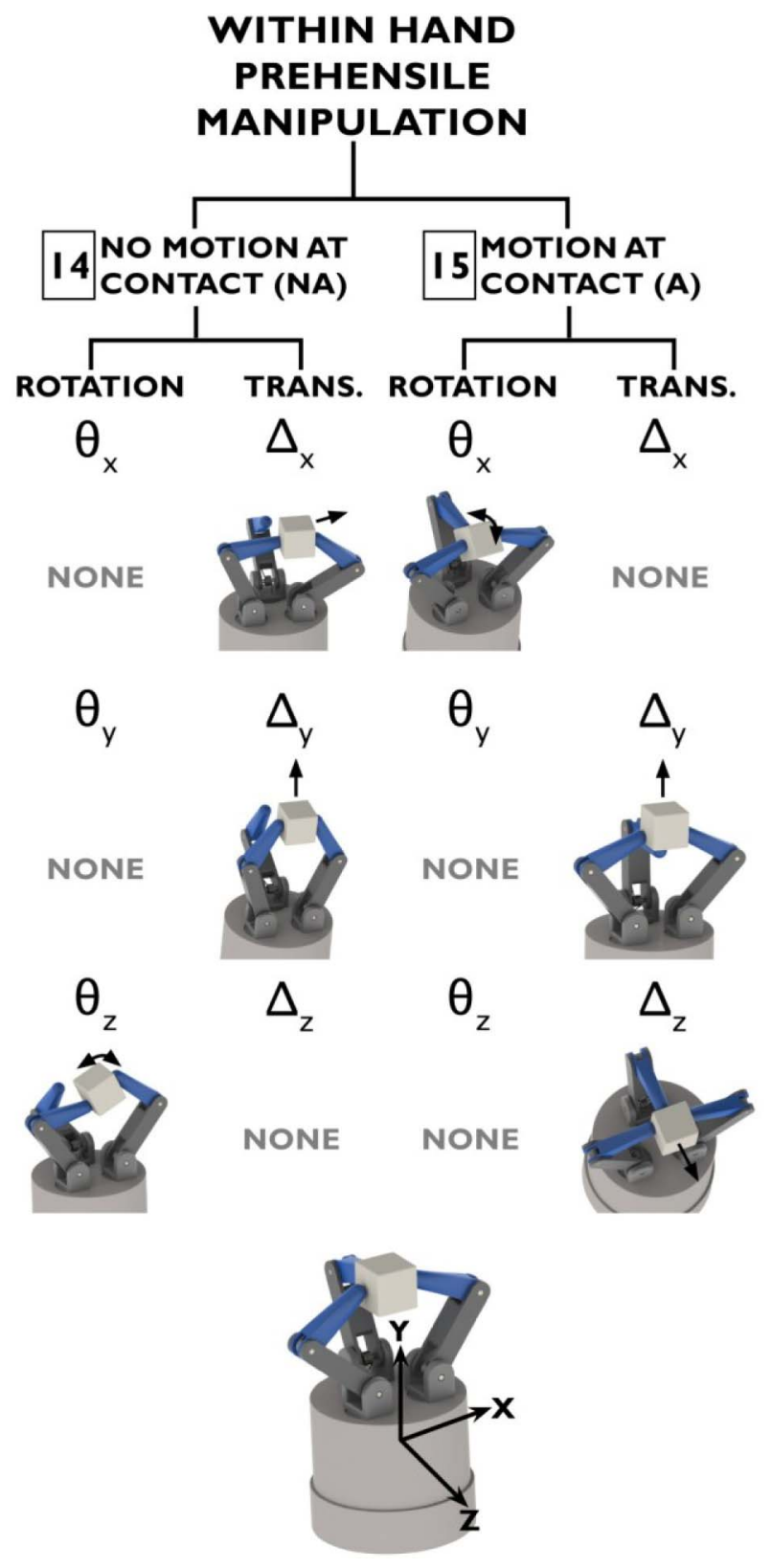

Fig. 11. Dexterous subclassification analysis for a simple three-finger robot hand. This example hand has two revolute degrees of freedom in each of three fingers, but is not able to rotate the two-link fingers at the base. As a result, within-hand manipulation is possible only along or around certain axes.

for a set of tasks for the purposes of preventing repetitive strain injuries. This type of analysis may be particularly beneficial in certain domains, such as assistive devices and design for manual assembly.

Many assistive devices are designed to help make tricky dexterous tasks easier for people with hand impairments. For example, buttoning the cuffs on a dress shirt is essentially impossible to accomplish without employing very dexterous in-hand manipulative movements and cannot be done by amputees with prosthetic terminal devices. Accordingly, one can purchase specially made button puller device to make buttoning clothing require less dexterous movements, and less within-hand movements. By observing users of a potential assistive device and applying the taxonomy, one can establish quantitatively whether the device is successful in reducing the task dexterity required.

Design for manual assembly is a domain, where parts of a device are optimized for efficient manipulation into their final configuration. Significant work has gone into estimating how different properties of the parts in a device affect the ease of assembly [45]. The proposed taxonomy could be used during the observation of an assembly task to assess which stages of assembly require high levels of dexterity, as evidenced by within hand and bimanual manipulation in a variety of directions of motion. This analysis could then be used to redesign problem parts for quicker overall assembly.

\subsection{Manipulation during ADLs}

The manipulation classification can also be used to compare the dexterous manipulation required to accomplish important ADLs. Fig. 12 shows the time-varying classification for three ADLs: an eating/drinking task, a transportation task, and a clothing management task. These tasks were recorded using a head mounted fisheye camera system [31] to avoid disturbing natural manipulation motions.

Three tasks were recorded to provide a variety of different ADL types with different dexterity requirements. The first task, taking a drink, involved pouring water from a pitcher into a coffee mug, and then taking a single sip from the mug. The second task, opening a door, involved removing a key ring with several keys from a jeans pocket, selecting the correct key, and then opening a door. The final task, putting on socks, involved putting on a pair of socks, starting with both socks in one hand and ending with the researcher standing up.

The time-varying representation of the manipulation classification demonstrates marked differences between the tasks. The drink task is shown to involve very little withinhand manipulation, and once water is in the cup, the left hand is not used at all while the final sip is taken. The door task shows a much different pattern. The left hand is used dexterously from the start to manipulate the keys. The right hand is used to help select the correct key and then the keys are quickly swapped between hands (note the loss of left hand contact around $8 \mathrm{~s}$ ). Finally, the right hand is used in a nonprehensile capacity to push the door open, while the left hand continues to perform dexterous key turning and key extraction motions. In the final socks task, both hands have contact and motion for almost the entire task.

In addition to considering the time-varying manipulation transitions graphically, various summary statistics can be used to further quantify the characteristics of a task. Table 3 provides several examples. A simple starting point is to calculate proportions of the task duration spent with each of the manipulation criteria active. In this case, we see that there is a high proportion of within-hand manipulation for both hands with the socks task and one hand for the door task, while for the drink task there is little within-hand activity. The prehensile proportion is fairly balanced for the drink and socks tasks, but unbalanced in the door task, reflecting a nonprehensile push which was used by one hand during the door task. 
Taking a drink
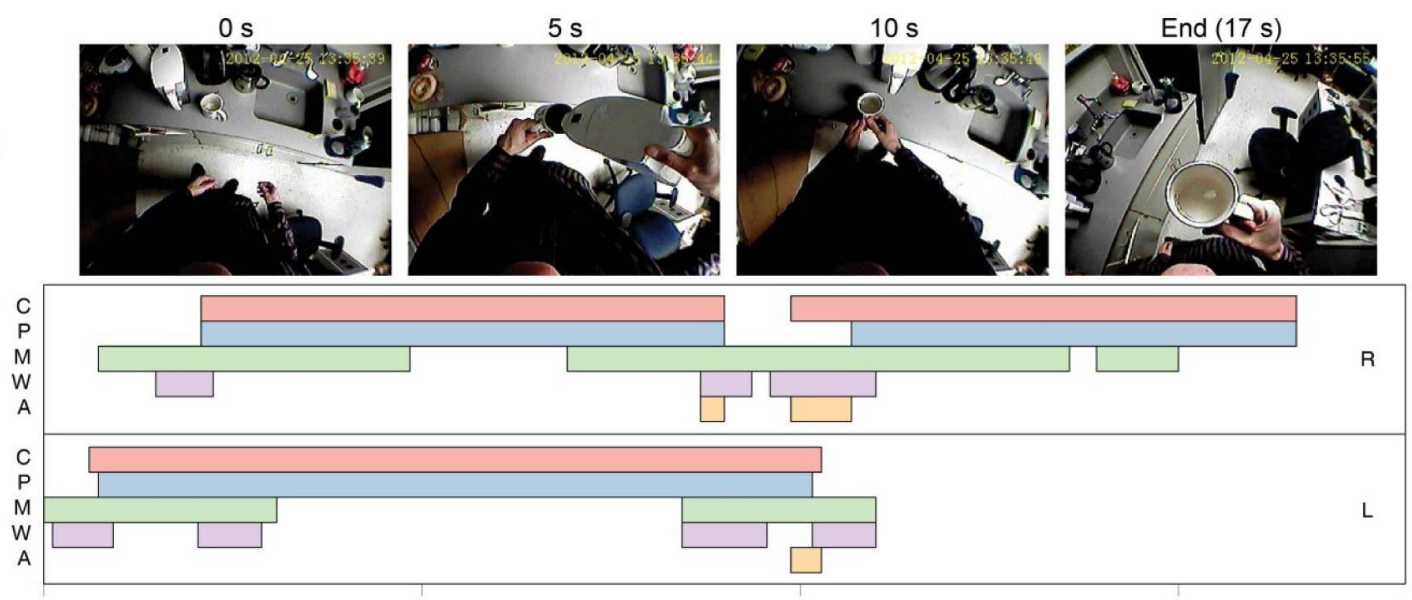

0

5

10

15

Time (s)

Opening a door

$0 \mathrm{~s}$ $5 \mathrm{~s}$

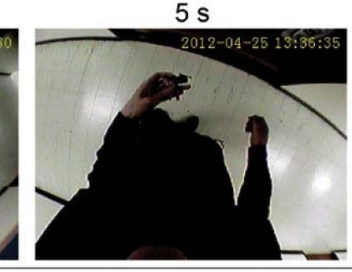

$10 \mathrm{~s}$

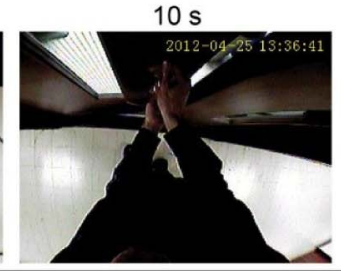

End $(13 \mathrm{~s})$
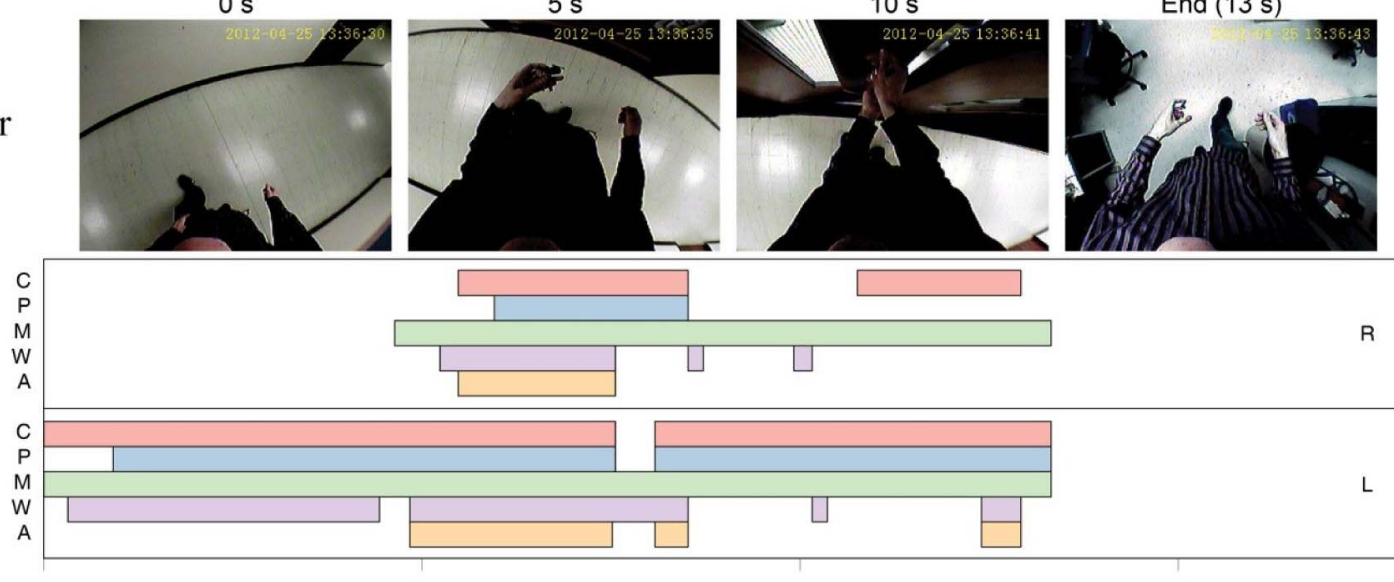

5

15

Time (s)
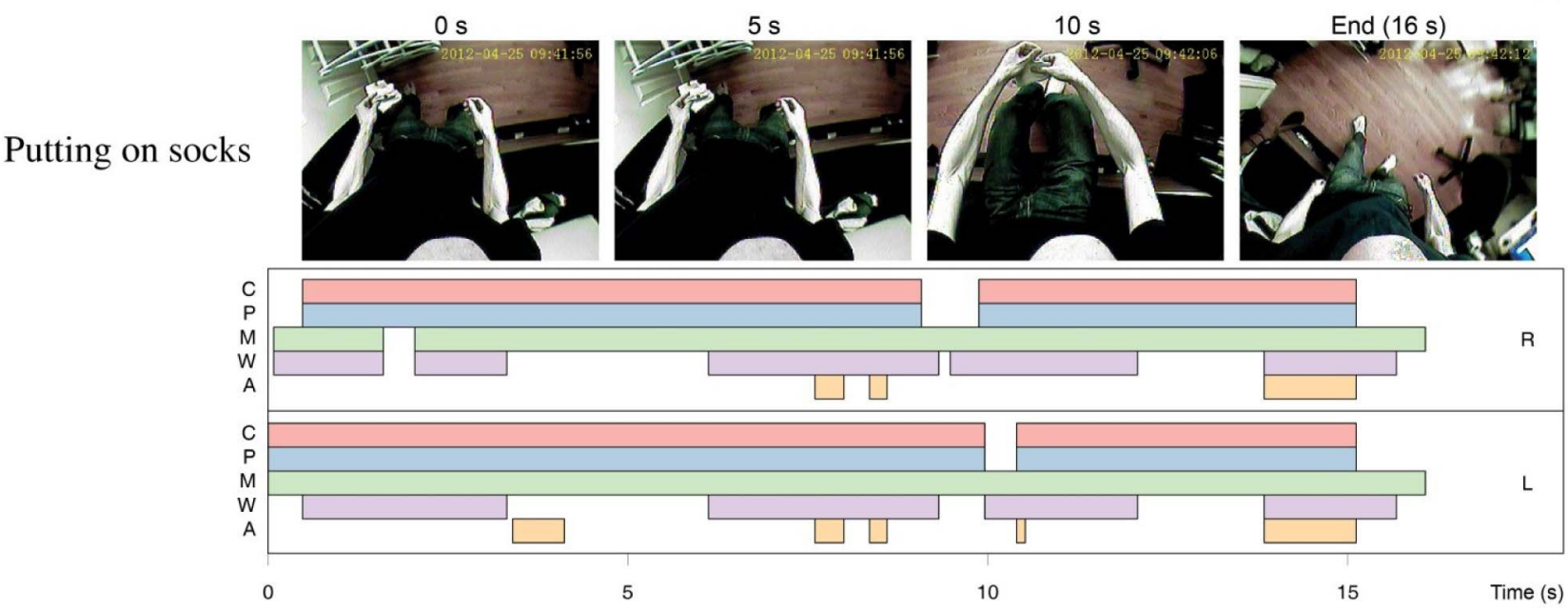

Fig. 12. Manipulation classification transitions for the three ADLs analyzed. In each plot, the top portion labeled "R" is for the right hand, and the bottom "L" portion is the left hand. The letters C, P, M, W, and A are abbreviations for contact, prehensile, motion, within-hand motion, and motion and contact, respectively. The drink task involved pouring water from a pitcher into a coffee mug and taking a single sip from the mug. The door task was to take keys out of a pants pocket, select the correct key, and open a door. The socks task began with two socks in one hand and ended when the subject stood up after placing both socks on their feet.

Statistics can also be calculated based on the axes of object motion observed during task execution. If we consider the proportions of translation and rotation during the ADLs, we see that they are fairly balanced during the door and drink task, while much more translation is used during the socks task. This manipulation pattern results because during the socks task, the sock edges were often put in a simple lateral pinch by both hands and then translated as needed to open up the socks and to pull the socks over the feet. The translation and rotation motions can also be analyzed in terms of their individual axes, showing, for example, the large amount of distal/proximal translation during the socks 
TABLE 3

Summary Statistics for the Three ADL Tasks

\begin{tabular}{|c|c|c|c|}
\hline Summary Statistic & \multicolumn{3}{|c|}{ Task } \\
\hline & Drink & Door & Socks \\
\hline Task duration (s) & 16.56 & 13.32 & 16.48 \\
\hline Classification changes per second & 2.23 & 2.10 & 2.06 \\
\hline Left contact proportion & 0.58 & 0.96 & 0.89 \\
\hline Right contact proportion & 0.82 & 0.39 & 0.84 \\
\hline Left prehensile proportion & 0.57 & 0.89 & 0.89 \\
\hline Right prehensile proportion & 0.77 & 0.19 & 0.84 \\
\hline Left motion proportion & 0.34 & 1.00 & 0.98 \\
\hline Right motion proportion & 0.71 & 0.65 & 0.94 \\
\hline Left within hand proportion & 0.22 & 0.64 & 0.61 \\
\hline Right within hand proportion & 0.17 & 0.21 & 0.63 \\
\hline Left motion at contact proportion & 0.02 & 0.27 & 0.17 \\
\hline Right motion at contact proportion & 0.07 & 0.16 & 0.12 \\
\hline Translation proportion & 0.28 & 0.44 & 0.82 \\
\hline Rotation proportion & 0.39 & 0.35 & 0.09 \\
\hline Radial/ulnar translation proportion & 0.08 & 0.03 & 0.13 \\
\hline Distal/proximal translation proportion & 0.14 & 0.34 & 0.56 \\
\hline Dorsal/palmar translation proportion & 0.07 & 0.06 & 0.14 \\
\hline Radial/ulnar rotation proportion & 0.10 & 0.04 & 0.03 \\
\hline Distal/proximal rotation proportion & 0.14 & 0.28 & 0.05 \\
\hline Dorsal/palmar rotation proportion & 0.14 & 0.04 & 0.00 \\
\hline $\begin{array}{l}\text { Bimanual simultaneous within hand } \\
\text { proportion }\end{array}$ & 0.10 & 0.17 & 0.58 \\
\hline Bimanual symmetric motion proportion & 0.00 & 0.09 & 0.63 \\
\hline
\end{tabular}

task as the socks were lowered down below the feet and then pulled back up over the feet.

Finally, the summary statistics can be used to show the type of bimanual manipulation occurring. The proportion of the task duration spent with within-hand manipulation occurring in both hands was calculated, showing the socks and door task to require the dexterous capabilities of both hands. In addition, a bimanual symmetric proportion was calculated based off the proportion of the time that both hand are producing the same object motion relative to their respective axes. This statistic reveals the coordinated, symmetric manipulation patterns used in the execution of the sock task.

These general principles and analysis techniques could be extended much further. For example, by using the same analysis with a larger sampling of subjects and tasks, we could determine which ADLs require large proportions of within-hand manipulation or even bimanual within-hand manipulation, as well as which motions are required by each task. This information could then be used to identify tasks that persons with a specific impairment will not likely be able to perform, and appropriate steps taken (e.g., providing them with an assistive device for the application). Similarly, persons with a specific deficit (e.g., stroke) might be examined doing a specific task to identify, where they deviate from the typical pattern, identifying, where they are exhibiting problematic compensatory movements. A similar process might also be used to assess manipulation strategies that cannot be performed with a simple prosthesis such as the common split hook. Specifically, if the typical strategy used to accomplish a given manipulation task involves extensive within-hand motion, the task will likely be difficult for users of a split hook prosthesis to accomplish, and solutions such as additional assistive devices should be procured for the patient.

A detailed analysis of task data could also assist hand design engineers to optimize hand functionality for a given level of mechanical complexity. As shown in Section 5.2, the taxonomy can be used as a framework to analyze which types of motion a robotic system can perform, and then these robotic capabilities can be compared to the target human manipulation behavior from the analyzed task data. For example, if it was observed in the human ADL data set that humans frequently use within-hand distal-proximal translations, then the robot hand design could be optimized for these motions, while trading off less used motions. The data could also be used to decide the amount of dexterity to place in the hand rather than in the wrist or arm (see Section 3.2)-if a particular axis of motion occurs much more often without within-hand motion, then it could be optimized for in the wrist or arm design rather than in the hand design.

The classifications and summary statistics for a large set of human tasks could also be used to choose a smaller set of representative benchmarking tasks. The tasks would be chosen to span a similar proportion of different types of manipulation behavior. For this application, pairing the taxonomy with an object classification would help ensure that a suitable range of objects is also present in the benchmarking task set. This benchmarking task set could then be quite valuable for comparing the performance of robotic manipulation systems or prosthetic hands and setting clear design goals for these devices.

\section{Discussion}

\subsection{Relationship to Common Manipulation Terminology}

There have been a number of terms used previously in the literature to describe various subclasses of dexterous manipulation, often without clear definitions of their meaning. For time-separated motions, two common terms are regrasping [43] and finger gaiting [46]. Both of these tasks are used to overcome the kinematic limitations of a manipulation system for a particular task and consist of sequences of simpler movements. "Regrasping" involves releasing the grasped object (onto a table, for instance) and then grasping it in another configuration to change its position and orientation within the grasp. 


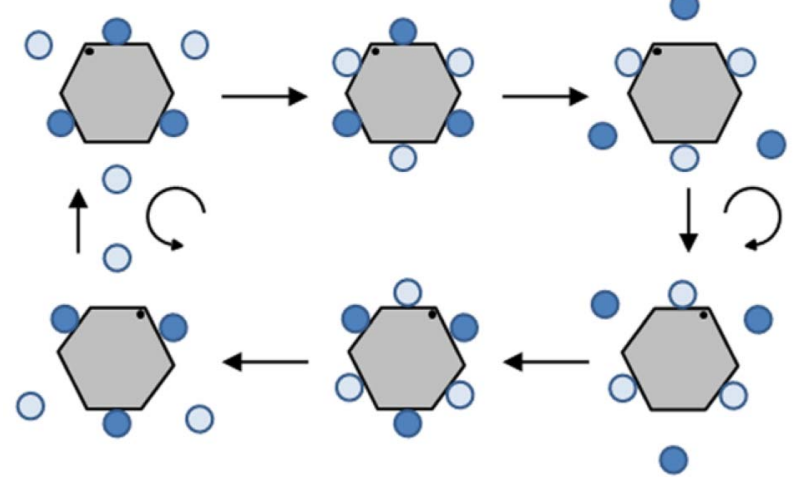

Fig. 13. Example of finger placement in finger gaiting.

A similar type of manipulation that is often also described as "regrasping" is "gaiting" or "finger gaiting." In these, the set of constraining contacts are exchanged typically within the hand, where grasping fingers are replaced once they have reached joint limits by free fingers with workspaces that can continue the motion (Fig. 13). Finger gaiting can be subdivided into finger substitution, where a free finger replaces a grasping finger at the edge of its configuration space, and finger rewind, where a free finger is used to maintain stability of the object while a grasping finger is freed to move to another position in its configuration space [46].

Other terminology has been used to describe discrete manipulation movements, each of which maps directly to one of the subclasses identified in our taxonomy, albeit sometimes with additional detail/specificity. Within-grasp manipulation [47] involves making small changes to the object's orientation and/or position while maintaining fingertip contact with the object and falls within the C P M W NA 14 subclass in our taxonomy. Pivoting/tracking [48] establishes an axis of object rotation through two point contacts while utilizing the remaining free fingers to guide the object's rotation about this axis (Fig. 14) and falls within the C P M W A 15 subclass in our taxonomy. Rolling [17], [49] is only realizable for objects/fingers of certain geometries, but can also be performed by nonfingered end-effectors. In rolling motions, one or more contact points moves across the surface of the object continuously, where no slip occurs between the object and manipulating surfaces, and falls within the C P M W A 15 class. Sliding [50] involves manipulating objects through controlled slip and is a more complicated version of push grasping [51]. Similar to rolling, it also falls within the C P M W A 15 class.

\subsection{Limitations}

While the authors believe the proposed taxonomy can be used to describe the high-level behavior of a hand during a manipulation task, and in this way can serve as a top-level taxonomy from which additional detail can be specified through subcategorizations. As such, it does not capture many of the low-level details about the hand configuration or the object being interacted with. This type of additional detail might be given through further subclassification of our taxonomy or by pairing it with another taxonomy.

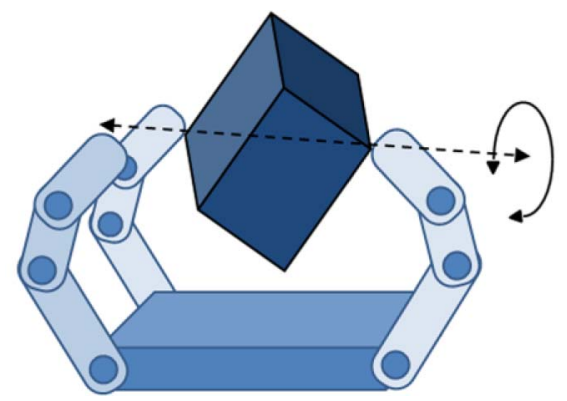

Fig. 14. Finger pivoting.

Further information related to the object being interacted with might be given by pairing the hand-centric taxonomy with an object-centric one (such as [37]) to relate the coordinate frames of the hand and its motion to the object motion. There may also be cases in which a description of the forces imparted by the hand (such as in [40]) is a useful addition.

To provide greater detail about the kinematic configuration of the hand during the manipulation movements, a grasp taxonomy such as [27] might be applied, the combination of which could be used to specify the shape of the hand during a given task. This approach might also allow one to analyze the types of manipulation associated with hand configurations (or grasp types). Applying this type of taxonomy would, however, be particular to a certain type of hand (such as a human or anthropomorphic hand) and would reduce some of the generality of the current manipulation taxonomy.

\subsection{Conclusions}

This work has considered dexterity and dexterous manipulation from both a human and robotic perspective. Various definitions for dexterity were presented, along with previous work on classifying manipulation. We discussed the challenges of distinguishing between arm and hand, and then argued that within-hand dexterity can offer expanded manipulation workspace and improved precision with reduced energy consumption, but at the cost of added complexity.

Following this discussion, our proposed classification scheme for hand-based manipulation was presented, which applies to both human and robot domains. Simple criteria were discussed that can be applied together to easily classify a wide range of manipulation behavior for any system in which a hand can be defined. The hand- and motion-centric structure of the taxonomy helps emphasize differences in hand function between different manipulation tasks and strategies.

Various applications of the taxonomy in the robot and human domains were then discussed. The taxonomy can be used to analyze manipulation strategies, as was done with the human coin pickup task. Following this analysis, the human strategy can be implemented in a robotic system, with appropriate modifications made to suit the particular robot architecture. The taxonomy can also be used to analyze the dexterous capabilities of a robot or prosthetic hand in terms of ability to produce different types of within-hand motion, or to better design products and parts 
for efficient human manipulation. Finally, the taxonomy can be used to examine the types of dexterity and patterns of manipulation used during various ADLs. This ADL analysis, if expanded, could be used to assess unusual manipulation patterns in impaired patients, to predict problem tasks for a specific impairment, and even to create a representative set of benchmarking tasks to give clear design goals and performance evaluation methods for a robotic or prosthetic hand design.

After this discussion of applications, important robotic manipulation terms were connected to the proposed classification scheme, followed by an appraisal of limitations of the taxonomy. Many of these limitations can be addressed by combining our taxonomy with other classification schemes such as hand configuration descriptions or force taxonomies, to provide additional detail. Overall, we believe our taxonomy provides a useful framework to describe hand usage during dexterous manipulation in a variety of different domains.

\section{ACKNOWLEDGMENTS}

This work was supported in part by the National Science Foundation Grant IIS-0953856 and US Defense Advanced Research Projects Agency Grant W91CRB-10-C-014. Preliminary versions of this paper were presented at the 2011 International Conference on Rehabilitation Robotics [52] and the 2011 International Conference on Advanced Robotics [53]. The authors would like to thank Lael Odhner, Josh Zheng, Leif Jentoft, Robert D. Howe, Joe Belter, and Sara de la Rosa for their helpful discussions related to the concepts presented in this paper, and to Kayla Matheus for helping to create some of the hand drawings used in the figures.

\section{REFERENCES}

[1] A. Faisal, D. Stout, J. Apel, and B. Bradley, “The Manipulative Complexity of Lower Paleolithic Stone Toolmaking," PloS One, vol. 5, no. 11, pp. 1-11, Jan. 2010.

[2] J.M. Hollerbach, "Workshop on the Design and Control of Dextrous Hands," AI Memo No. 661, 1982.

[3] P. Wright, J. Demmel, and M. Nagurka, "The Dexterity of Manufacturing Hands," Robotics Research, DSC, vol. 14, pp. 157163, 1989.

[4] A. Okamura, N. Smaby, and M.R. Cutkosky, "An Overview of Dexterous Manipulation," Proc. IEEE Int'l Conf. Robotics and Automation (ICRA), pp. 255-261, Apr. 2000.

[5] A. Bicchi, "Hands for Dexterous Manipulation and Robust Grasping: A Difficult Road toward Simplicity," IEEE Trans. Robotics and Automation, vol. 16, no. 6, pp. 652-662, Dec. 2000.

[6] A. Bicchi and A. Marigo, "Dexterous Grippers: Putting Nonholonomy to Work for Fine Manipulation," Int'l J. Robotics Research, vol. 21, no. 5/6, pp. 427-442, May 2002.

[7] Z. Li, J.F. Canny, and S.S. Sastry, "On Motion Planning for Dexterous Manipulation. I. The Problem Formulation," Proc. Int'l Conf. Robotics and Automation, no. 3, pp. 775-780, 1989.

[8] C.A. Klein and B.E. Blaho, "Dexterity Measures for the Design and Control of Kinematically Redundant Manipulators," Int'l J. Robotics Research, vol. 6, no. 2, pp. 72-83, June 1987.

[9] R.H. Sturges, "A Quantification of Machine Dexterity Applied to an Assembly Task," Int'l J. Robotics Research, vol. 9, no. 3, pp. 49-62, June 1990.

[10] S.C. Jacobsen, J.E. Wood, D.F. Knutti, and K.B. Biggers, "The UTAH/M.I.T. Dextrous Hand: Work in Progress," Int'l J. Robotics Research, vol. 3, no. 4, pp. 21-50, Dec. 1984.
[11] L. Bologni, S. Caselli, and C. Melchiorri, "Design Issues for the UB Robotic Hand," Proc. NATO Advanced Research Workshop Robots with Redundancy, 1988.

[12] F. Lotti, P. Tiezzi, G. Vassura, L. Biagiotti, and C. Melchiorri, "UBH 3: An Anthropomorphic Hand with Simplified EndoSkeletal Structure and Soft Continuous Fingerpads," Proc. IEEE Int'l Conf. Robotics and Automation, 2004.

[13] T. Mouri, H. Kawasaki, K. Yoshikawa, J. Takai, and S. Ito, "Anthropomorphic Robot Hand: Gifu Hand III," Proc. Int'l Conf. Control, Automation and Systems (ICCAS '02), pp. 1288-1293, 2002.

[14] J. Butterfaß, M. Grebenstein, H. Liu, and G. Hirzinger, "DLRHand II: Next Generation of a Dextrous Robot Hand," Proc. IEEE Int'l Conf. Robotics and Automation (ICRA), vol. 1, pp. 109-114, 2001.

[15] C.S. Lovchik and M.a. Diftler, "The Robonaut Hand: A Dexterous Robot Hand for Space," Proc. IEEE Int'l Conf. Robotics and Automation, vol. 2, pp. 907-912, May 1999.

[16] N. Fukaya, S. Toyama, T. Asfour, and R. Dillmann, "Design of the TUAT/Karlsruhe Humanoid Hand," Proc. IEEE/RSJ Int'l Conf. Intelligent Robots and Systems, vol. 3, pp. 1754-1759, 2000.

[17] A. Bicchi and R. Sorrentino, "Dexterous Manipulation through Rolling," Proc. IEEE Int'l Conf. Robotics and Automation, vol. 1, pp. 452-457, 1995.

[18] K. Nagata, "Manipulation by a Parallel-Jaw Gripper Having a Turntable at Each Fingertip," Proc. IEEE Int'l Conf. Robotics and Automation, pp. 1663-1670, 1994.

[19] R. Balasubramanian, B. Dellon, S. Pradhan, S. Gibbs, and Y. Matsuoka, "Quantifying the Dimensions of Human Hand Dexterity through a Survey of Daily Tasks," Proc. Workshop Understanding the Human Hand for Advancing Robotic Manipulation at Robotics: Science and Systems, 2009.

[20] M.L. Latash, M.T. Turvey, and N.A. Bernstein, Dexterity and Its Development. Lawrence Erlbaum, 1996.

[21] K.E. Yancosek and D. Howell, "A Narrative Review of Dexterity Assessments," J. Hand Therapy, vol. 22, no. 3, pp. 258-269, 2009.

[22] D.A. Nowak, "Toward an Objective Quantification of Impaired Manual Dexterity Following Stroke: The Usefulness of Precision Grip Measures," Clinical Neurophysiology, vol. 117, no. 7, pp. 1409-11, July 2006.

[23] M.E. Williams, N.M. Hadler, and J.A.L. Earp, "Manual Ability as a Marker of Dependency in Geriatric Women," J. Chronic Diseases, vol. 35, no. 2, pp. 115-22, Feb. 1982.

[24] C. Metcalf, B.A. Hons, J. Adams, J. Burridge, V. Yule, and P. Chappell, "A Review of Clinical Upper Limb Assessments within the Framework of the WHO ICF," Musculoskeletal Care, vol. 5, no. 3, pp. 160-173, 2007.

[25] M. Fluet, O. Lambercy, and R. Gassert, "Upper Limb Assessment Using a Virtual Peg Insertion Test," Proc. Int'l Conf. Rehabilitation Robotics (ICORR), 2011.

[26] T. Iberall, "Human Prehension and Dexterous Robot Hands," The Int'l J. Robotics Research, vol. 16, no. 3, pp. 285-299, June 1997.

[27] T. Feix, R. Pawlik, H.-B. Schmiedmayer, J. Romero, and D. Kragic, "A Comprehensive Grasp Taxonomy," Proc. Robotics, Science and Systems Conf.: Workshop Understanding the Human Hand for Advancing Robotic Manipulation: Poster Presentation, 2009.

[28] G. Schlesinger, "Der Mechanische Aufbau Der Kunstlichen Glieder," Ersatzglieder und Arbeitshilfen für Kriegsbeschädigte und Unfallverletzte, pp. 321-661. Springer, 1919.

[29] J.R. Napier, "The Prehensile Movements of the Human Hand," J. Bone and Joint Surgery, vol. 38, no. 4, pp. 902-913, 1956.

[30] M. Cutkosky and R.D. Howe, "Human Grasp Choice and Robotic Grasp Analysis," Dextrous Robot Hands, pp. 5-31, Springer-Verlag, 1990.

[31] J. Zheng, S. De La Rosa, and A. Dollar, "An Investigation of Grasp Type and Frequency in Daily Household and Machine Shop Tasks," Proc. Int'l Conf. Robotics and Automation, pp. 4169-4175, 2011.

[32] M. Santello, M. Flanders, and J.F. Soechting, "Postural Hand Synergies for Tool Use," J. Neuroscience, vol. 18, no. 23, pp. $10105-$ 15, Dec. 1998.

[33] J.M. Elliott and K. Connolly, "A Classification of Manipulative Hand Movements," Developmental Medicine \& Child Neurology, vol. 26, no. 3, pp. 283-296, 1984.

[34] C.E. Exner, "In-Hand Manipulation Skills," Development of Hand Skills in the Child, pp. 35-45, Am. Occupational Therapy Assoc., 1992. 
[35] J.H. Carr, R.B. Shepherd, J. Gordon, A.M. Gentile, and J.M. Held "Skill Acquisition: Action, Movement, and Neuromotor Processes," Movement Science: Foundations for Physical Therapy in Rehabilitation, pp. 93-149, Aspen, 1987.

[36] T.H. Speeter, "Primitive Based Control of the Utah/MIT Dextrous Hand," Proc. IEEE Int'l Conf. Robotics and Automation, pp. 866-877, Apr. 1991.

[37] G. Morris and L. Haynes, "Robotic Assembly by Constraints," Proc. IEEE Int'l Conf. Robotics and Automation, pp. 1507-1515, 1987.

[38] J.D. Morrow and P.K. Khosla, "Manipulation Task Primitives for Composing Robot Skills," Proc. Int'l Conf. Robotics and Automation, pp. 3354-3359, 1997.

[39] P. Michelman, "Precision Object Manipulation with a Multifingered Robot Hand," IEEE Trans. Robotics and Automation, vol. 14, no. 1, pp. 105-113, Feb. 1998.

[40] A. Bloomfield, Y. Deng, J. Wampler, P. Rondot, D. Harth, M. McManus, and N. Badler, "A Taxonomy and Comparison of Haptic Actions for Disassembly Tasks," Proc. IEEE Virtual Reality, 2003.

[41] J.S. Pettinato and H.E. Stephanou, "Manipulability and Stability of a Tentacle Based Robot Manipulator," Proc., Int'l Conf. Robotics and Automation, pp. 458-463, 1989.

[42] S. Hart, S. Sen, and R. Grupen, "Generalization and Transfer in Robot Control," Proc. Epigenetic Robotics Ann. Conf., 2008.

[43] P. Tournassoud, T. Lozano-Perez, and E. Mazer, "Regrasping," Proc. IEEE Int'l Conf. Robotics and Automation, vol. 4, pp. 1924-1928, 1987.

[44] L.U. Odhner, R.R. Ma, and A.M. Dollar, "Precision Grasping and Manipulation of Small Objects from Flat Surfaces Using Underactuated Fingers," Proc. IEEE Int'l Conf. Robotics and Automation (ICRA), 2012.

[45] G. Boothroyd, P. Dewhurst, and W.A. Knight, Product Design for Manufacture and Assembly, vol. 26, no. 7, pp. 505-520. CRC Press, 2001.

[46] L. Han and J.C. Trinkle, "Dextrous Manipulation by Rolling and Finger Gaiting," Proc. IEEE Int'l Conf. Robotics and Automation, vol. 1, pp. 730-735, May 1998

[47] D. Osswald, "Mechanical System and Control System of a Dexterous Robot Hand," Proc. IEEE-RAS Int'l Conf. Humanoid Robots, 2001.

[48] D. Rus, "Dexterous Rotations of Polyhedra," Proc. IEEE Int'l Conf. Robotics and Automation, pp. 2758-2763, 1992.

[49] L. Han, Y. Guan, Z. Li, Q. Shi, and J. Trinkle, "Dextrous Manipulation with Rolling Contacts," Proc. IEEE Int'l Conf. Robotics and Automation, vol. 2, pp. 992-997, Apr. 1997.

[50] D.L. Brock, "Enhancing the Dexterity of a Robot Hand Using Controlled Slip," Proc. IEEE Int'l Conf. Robotics and Automation, no. 7, pp. 249-251, 1988.

[51] M.R. Dogar and S.S. Srinivasa, "Push-Grasping with Dexterous Hands: Mechanics and a Method," Proc. IEEE/RSJ Int'l Conf. Intelligent Robots and Systems (IROS), pp. 2123-2130, 2010.

[52] I.M. Bullock and A.M. Dollar, "Classifying Human Manipulation Behavior," Proc. IEEE Int'l Conf. Rehabilitation Robotics, 2011.

[53] R.R. Ma and A.M. Dollar, "On Dexterity and Dexterous Manipulation," Proc. 15th Int'l Conf. Advanced Robotics, 2011.

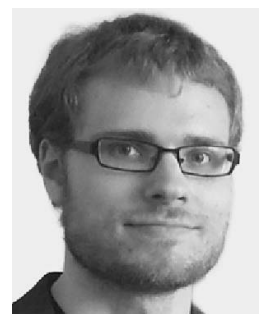

Ian M. Bullock received the BS degree in engineering from Harvey Mudd College, Claremont, California, in 2010 and started working toward the $\mathrm{PhD}$ degree at Yale University in the fall of 2010. His work experience has ranged from programming educational neuroscience modules to designing circuits for a particle accelerator. His research interests include human dexterity and robotic manipulation, with particular emphasis on using an understanding of the capabilities of the human hand to improve the design of robotic and prosthetic manipulators. He is a student member of the IEEE.

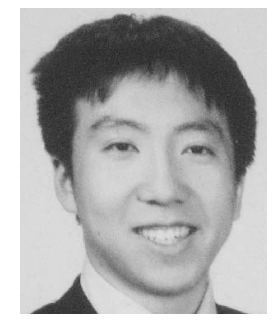

Raymond $\mathbf{R}$. Ma received the BS degree in mechanical engineering and the BS degree in computer science from the Massachusetts Institute of Technology in 2010 and is currently working toward the $\mathrm{PhD}$ degree in engineering at Yale University. His research focuses on the dexterous capabilities of compliant, underactuated robotic graspers. $\mathrm{He}$ is a student member of the IEEE.

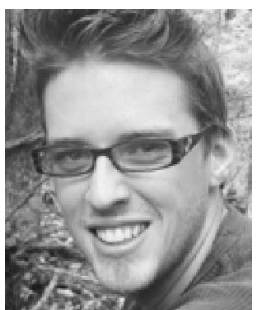

Aaron M. Dollar received the BS degree in mechanical engineering from the University of Massachusetts at Amherst, the SM and PhD degrees in engineering sciences from Harvard, and conducted two years of postdoctoral research at the MIT Media Lab. He has been an assistant professor of mechanical engineering and materials science at Yale since January 2009. His research interests include human and robotic grasping and dexterous manipulation, mechanisms and machine design, and assistive and rehabilitation devices including upper limb prosthetics and lower limb orthoses. He is the cofounder and editor of RoboticsCourseWare.org, an open repository for robotics pedagogical materials. He was the recipient of the 2011 Young Investigator Award from the Air Force Office of Scientific Research, the 2010 Technology Review TR35 Young Innovator Award, and the 2010 National Science Foundation CAREER Award. $\mathrm{He}$ is a member of the IEEE.

$\triangleright$ For more information on this or any other computing topic, please visit our Digital Library at www.computer.org/publications/dlib. 